\title{
Acknowledgement of editors and reviewers
}

Received: 9 January 2013 / Accepted: 14 January 2013 /Published online: 24 January 2013

(C) Springer Science+Business Media Dordrecht and International Society for Plant Pathology 2013

I should like to thank the Senior Editors and Associate Editors of the journal as well as the following reviewers who have generously given up valuable time during 2012 to review papers for Food Security. The success of the journal depends upon their care and competence. Their conscientiousness is much appreciated.

Richard Strange Editor-in-Chief, Food Security

1. Aase, Tor, Dept of Geography, University of Bergen, NORWAY

2. Adimassu, Zenebe, Ethiopian Institute of Agricultural Research, ETHIOPIA

3. Afari-Sefa, Victor, Regional Center for Africa, AVRDC-The World Vegetable Center, TANZANIA

4. Akhtar-Schuster, Mariam, GERMANY

5. Alam, Ferdous, Institute of Agricultural and Food Policy Studies, Universiti Putra Malaysia, MALAYSIA

6. Ames, Bruce, UNITED STATES

7. Anjum, Fauzia, Department of Chemistry, Drexel University, Chestnut Street, Philadelphia, UNITED STATES

8. Banson, Kwamina, Socio-Economics, Biotechnology and Nuclear Agriculture Research Institute, GHANA

9. Barnett, Jon, AUSTRALIA

10. Barrett, Christopher, Department of Applied Economics and Management, Cornell University, UNITED STATES

11. Battilani, Paola, ITALY

12. Bellemare, Marc, CANADA

13. Belton, Benjamin, BANGLADESH

14. Benson, Todd, UNITED STATES

15. Berkhout, Ezra, NETHERLANDS

16. Bidogeza, Jean-Claude, Natural Resource Management Theme, International Centre for Soil Fertility and Agricultural Development, KENYA

17. Block, Steven, UNITED STATES
18. Boyns, Ken, UNITED KINGDOM

19. Brown, Molly, Biospheric Sciences Branch, NASA Goddard Space Flight Center, UNITED STATES

20. Brownhill, Leigh, CANADA

21. Bryceson, Deborah, UNITED KINGDOM

22. Bunn, J, UNITED KINGDOM

23. Burger, Kees, NETHERLANDS

24. Byerlee, Derek, UNITED STATES

25. Chakrabarty, Sayan, BANGLADESH

26. Chant, Sylvia, UNITED KINGDOM

27. Chege, Ayub, UNITED KINGDOM

28. Christiaensen, Luc, BELGIUM

29. Coates, Jennifer, Friedman School of Nutrition Science and Policy, Tufts University, UNITED STATES

30. Corredera, Lourdes, Producción Animal y Ciencia de los Alimentos, Universidad de Zaragoza, SPAIN

31. Creamer, Bernardo, UNITED STATES

32. Dawe, David, UNITED STATES

33. de Graaff, Jan, Land Degradation and Development Group, Wageningen University, NETHERLANDS

34. De Pauw, Eddy, ICARDA, SYRIAN ARAB REPUBLIC

35. Deere, Carmen Diana, UNITED STATES

36. Deryng, Delphine, UNITED KINGDOM

37. Devereux, Stephen, UNITED KINGDOM

38. D'Haese, Marijke, BELGIUM 
39. Di Falco, Salvatore, London School of Economics, UNITED KINGDOM

40. Diagne, Mandiaye, Policy and Impact Assessment, Africa Rice Center (AfricaRice), SENEGAL

41. Dusunceli, Fazil, FAO, ITALY

42. Ericksen, Polly, PLE and SLF, ILRI, KENYA

43. Erskine, William, Centre for Legumes in Mediterranean Agriculture, The University of Western Australia, AUSTRALIA

44. Fabiansson, Stefan, ITALY

45. Fanzo, Jessica, REACH Interagency Partnership on Child Hunger and Undernutrition, UN World Food Programme, ITALY

46. Fleskens, Luuk, UNITED KINGDOM

47. Fraser, Evan, CANADA

48. Friday, James, UNITED STATES

49. Fullbrook, David, UNITED KINGDOM

50. Gajigo, Ousman, World Bank, UNITED STATES

51. Gali, Basavaraj, Dryland Cereals, ICRISAT, INDIA

52. Giller, Ken, NETHERLANDS

53. Glover, Dominic, NETHERLANDS

54. Gohin, Alexandre, FRANCE

55. Guthiga, Paul, KENYA

56. Haji, Jema, ETHIOPIA

57. Harttgen, Kenneth, GERMANY

58. Hayes, Daniel, UNITED STATES

59. Headey, Derek, UNITED STATES

60. Hellin, Jonathan, MEXICO

61. Hengsdijk, Huibe, NETHERLANDS

62. Hickey, Gordon, Natural Resource Sciences, McGill University, CANADA

63. Hoddinott, John, International Food Policy Research Institute, UNITED STATES

64. Hodges, Rick, UNITED KINGDOM

65. Howie, Charles, UNITED KINGDOM

66. Hsiaoping, Chien, JAPAN

67. Hundie, Bekele, Agriculture and Natural Resources, Ethiopian Economics Association/Ethiopian Economic Policy Research Institute, ETHIOPIA

68. Hussain, Abid, Regional and Rural Development Planning; School of Environment, Resources and Asian Institute of Technology, THAILAND

69. Ibnouf, Fatma, Development Studies and Research Institute, University of Khartoum, SUDAN

70. Jafry, Tahseen, UNITED KINGDOM

71. Johnson, Nancy, KENYA

72. Joshi, Krishna, NEPAL

73. Joshi, Arun, NEPAL

74. Kaliba, Aloyce, Department of Economics and Finance College of Business, Southern University and A\&M College, UNITED STATES

75. Kassie, Menale, KENYA

76. Kastner, Thomas, AUSTRIA
77. Kebede, Bereket, ETHIOPIA

78. Keding, Gudrun, Biodiversity, KENYA

79. Kolawole, Oluwatoyin, Okavango Research Institute, University of Botswana, BOTSWANA

80. Kosec, Katrina, UNITED STATES

81. Kuiper, Harry, Wageningen University, NETHERLANDS

82. Kuuire, Vincent Z., Geography, University of Western Ontario, CANADA

83. Kwena, Arthur, KENYA

84. Lane, David A., ITALY

85. Langyintuo, Augustine, UNITED STATES

86. Launay, Marie, FRANCE

87. Leathers, Howard, Department of Agricultural and Resource Economics, University of Maryland, UNITED STATES

88. Li, Xiaoyun, Huazhong Agricultural University; CIMMYT, CHINA

89. Mahuku, George, MEXICO

90. Mainardi, Stefano, Economics, University of Botswana, BOTSWANA

91. Mainuddin, Mohammed, AUSTRALIA

92. Mansfield, John, UNITED KINGDOM

93. Marenya, Paswel, KENYA

94. Matthews, Peter, UNITED STATES

95. McGuire, Shawn, University of East Anglia, School of International Development, UNITED KINGDOM

96. Miyasaka, Susan, UNITED STATES

97. Mkwambisi, David, MALAWI

98. Monyo, Emmanuel, MALAWI

99. Moretti, Antonio, Institute of Sciences of Food Production, National Research Council, ITALY

100. Mugera, Amin, AUSTRALIA

101. Mukherjee, Protap, INDIA

102. Nakamura, Satoshi, JAPAN

103. Naseem, Anwar, Department of Natural Resource Sciences, McGill University, CANADA

104. Obare, Gideon, KENYA

105. Oerke, E.C., GERMANY

106. Ohlander, Lars, SWEDEN

107. Okello, Julius J, Agricultural Economics, University of Nairobi, KENYA

108. Orchard, Steven, UNITED KINGDOM

109. Ortiz, Rodomiro, SWEDEN

110. Oswald, Andreas, ETHIOPIA

111. Ouma, James, KENYA

112. Page, Sam, UNITED KINGDOM

113. Pelletier, Bernard, CANADA

114. Pelletier, Nathan, BELGIUM

115. Pereira, Rosangela Alves, BRAZIL

116. Perry, Joe, UNITED KINGDOM

117. Phillips, Mike, MALAYSIA

118. Pridmore, Andrew, UNITED KINGDOM 
119. Qaim, Matin, Department of Agricultural Economics and Rural Development, Georg-August-University of Goettingen, GERMANY

120. Quatrini, Simone, ITALY

121. Rai, K, INDIA

122. Rao, NH, NAARM, INDIA

123. Rattan, Suresh, DENMARK

124. Richards, Paul, NETHERLANDS

125. Richardson, Robert, UNITED STATES

126. Rijkers, Ger, NETHERLANDS

127. Ritieni, Alberto, ITALY

128. Rocha, Cecilia, CANADA

129. Rogers, Beatrice, UNITED STATES

130. Rossi, Frederick, BANGLADESH

131. Safa, Helen, UNITED STATES

132. Sant'ana, Anderson, Department of Food and Experimental Nutrition, University of São Paulo, BRAZIL

133. Savary, Serge, AGIR, INRA, FRANCE

134. Shackleton, Charlie, SOUTH AFRICA

135. Sharma, Abhijit, UNITED KINGDOM

136. Shephard, Gordon, SOUTH AFRICA

137. Shiferaw, Bekele, Socio-Economics Program, CIMMYT International Maize and Wheat Improvement Center, KENYA

138. Shimokawa, Satoru, HONG KONG

139. Simelton, Elisabeth, UNITED KINGDOM

140. Singh, Kehar, Aquaculture/Fisheries Center, University of Arkansas at Pine Bluff, UNITED STATES

141. Skaggs, Rhonda, Agricultural Economics, New Mexico State University, UNITED STATES

142. Slingerland, Maja, NETHERLANDS

143. Smith, Monica, Anthropology, University of California - Los Angeles, UNITED STATES

144. Smith, Francisca, CANADA
145. Somerset, Shawn, AUSTRALIA

146. Speelman, Stijn, BELGIUM

147. Spielman, David, International Food Policy Research Institute, UNITED STATES

148. Strange, Richard, Birkbeck College, University of London UNITED KINGDOM

149. Suckall, Natalie, UNITED KINGDOM

150. Sudini, Harikishen, INDIA

151. Sumberg, James, UNITED KINGDOM

152. Tabuti, JRS, UGANDA

153. Tawodzera, Godfrey, African Centre for Cities (ACC), University of Cape Town, SOUTH AFRICA

154. Temme, L., NETHERLANDS

155. Teng, Paul, MALAYSIA

156. Thilmany, Dawn, UNITED STATES

157. Tiwari, Prakash, Geography, Kumaun University, Nainital, India, INDIA

158. Uphoff, Norman, UNITED STATES

159. Vagsholm, Ivar, SWEDEN

160. Vallino, Elena, Dipartimento di Scienze Economiche e Finanziarie, Università del Piemonte Orientale, ITALY

161. Vas, Janos, AUSTRIA

162. Vaughan, Duncan, JAPAN

163. Vegi, Anuradha, Veterinary and Microbiological Sciences, North Dakota State University, UNITED STATES

164. Waddington, Stephen, MEXICO

165. Wale, Edilegnaw, SOUTH AFRICA

166. Watanabe, Takeshi, Research Program - Resilient Dryland Systems, ICRISAT, INDIA

167. Wesenbeeck, van, Lia, NETHERLANDS

168. Zhang, Xu, UNITED KINGDOM

169. Zhu, Tingju, UNITED STATES 